\title{
Chiari Osteotomy Plus Shelf Procedure in Neuromuscular Hip Dysplasia
}

\author{
Bradley M Garcia ${ }^{1}$, Mike Scharf ${ }^{1}$, Andrew G Georgiadis ${ }^{2}$ and Mark A Seeley ${ }^{3}$ \\ ${ }^{1}$ Geisinger Commonwealth School of Medicine, USA \\ ${ }^{2}$ Gillette Children's Hospital, USA \\ ${ }^{3}$ Geisinger Medical Center, USA
}

\begin{abstract}
The management of pediatric neuromuscular hip dysplasia is complex and may involve reconstructive or salvage procedures based on the severity of the subluxation, underlying femoral head and acetabular deformity, skeletal maturity, and patient co-morbidities. We report the use of a Chiari osteotomy as salvage in the management of a case of advanced neuromuscular hip dysplasia. Surgical details of the osteotomy with shelf augmentation and three-year postoperative follow-up are included.
\end{abstract}

\section{Introduction}

The Chiari pelvic osteotomy [1] was first introduced as a salvage procedure by Karl Chiari in 1952 [2]; his osteotomy was indicated for congenital dislocations, dysplastic hip with osteoarthritis, coxa valga subluxans, coxa magna after Perthes disease, and paralytic hip in poliomyelitis or cerebral palsy [3]. The procedure is currently employed for adolescents and young adults who have painful hip subluxation and whose lack of femoral head coverage is severe with associated articular incongruity $[4,5]$.

One technical limitation of the osteotomy may be in providing adequate anterolateral coverage [6]. Therefore, a shelf augmentation can supplement the Chiari osteotomy if such coverage is desired [4]. The high rate of graft resorption with the shelf procedure makes this salvage combination a "last treatment option" (viz. for use in situations where hyper containment into the native acetabulum is unfeasible) $[4,7]$.

After adolescence, the incongruity and early osteoarthritis typically seen in individuals with neuromuscular hip dysplasia contraindicates a re-directional osteotomy of the acetabulum. In these cases, salvage procedures are viable treatment options to improve femoral head coverage, decrease stress on the joint, and prevent degenerative arthritis $[4,8]$. Severe spasticity may be a relative contraindication to joint arthroplasty in these cases. In individuals with neuromuscular hip dysplasia, both Chiari osteotomy and shelf augmentation can provide adequate femoral coverage without substantial limitations on hip mobility $[4,5,8]$.

A Chiari osteotomy aims to achieve lateral coverage of the femoral head and increase the weight bearing surface area of the hip joint by medializing the acetabulum through an intra-articular osteotomy. A transverse or slightly lateral-distal to medial-proximal osteotomy of the ilium is performed at the superior margin of the acetabulum. The osteotomy extends through the anterior and posterior columns of the pelvis and allows displacement of the acetabular fossa medially.
As most femoral heads are diseased from asymmetric wear, the superior capsule can be interposed between the acetabulum and femoral head and may need to be extirpated $[4,9,10]$. Ideally, the Chiari osteotomy is performed at an upward angle of $10^{\circ}$ and is shifted by $50 \%$ of the iliac width [4]. Displacing a Chiari osteotomy more than $50 \%$ may lead to delayed union [11]. Furthermore, a $20 \%$ reduction in the contact pressure on the femoral head can be achieved with a $15 \mathrm{~mm}$ Medialization [12].

We present a case of neuromuscular hip dysplasia with an incongruent hip joint and severe pain. While existing hip surveillance protocols in this patient population are intended to incite hyper containment treatment before reaching this clinical situation [13], the patient did not present to the authors' clinic until she had well progressed, advanced femoral head deformity with subluxation.

\section{Case Presentation}

A seventeen-year-old female functioning at a GMFCS IV level [14] presented to the clinic with her family with a chief complaint of increasing right sided hip pain over the antecedent four months. She had a past medical history significant for Antithrombin III Deficiency [15], which was responsible for a perinatal stroke and resultant spasticity and quadriplegic involvement. Cognitively the patient was able to communicate at a CFCS III level [16]. Prior to development of the hip pain, she was ambulatory with minimal assistance. Due to the progressive pain, she was now confined to a wheelchair. Perineal

*Corresponding author: Bradley M Garcia, Geisinger Commonwealth School of Medicine, Scranton, USA

Accepted: December 26, 2020

Published online: December 28, 2020

Citation: Garcia BM, Scharf M, Georgiadis AG, et al. (2020) Chiari Osteotomy Plus Shelf Procedure in Neuromuscular Hip Dysplasia. Adv Tech Musculoskelet Surg 4(2):50-54 

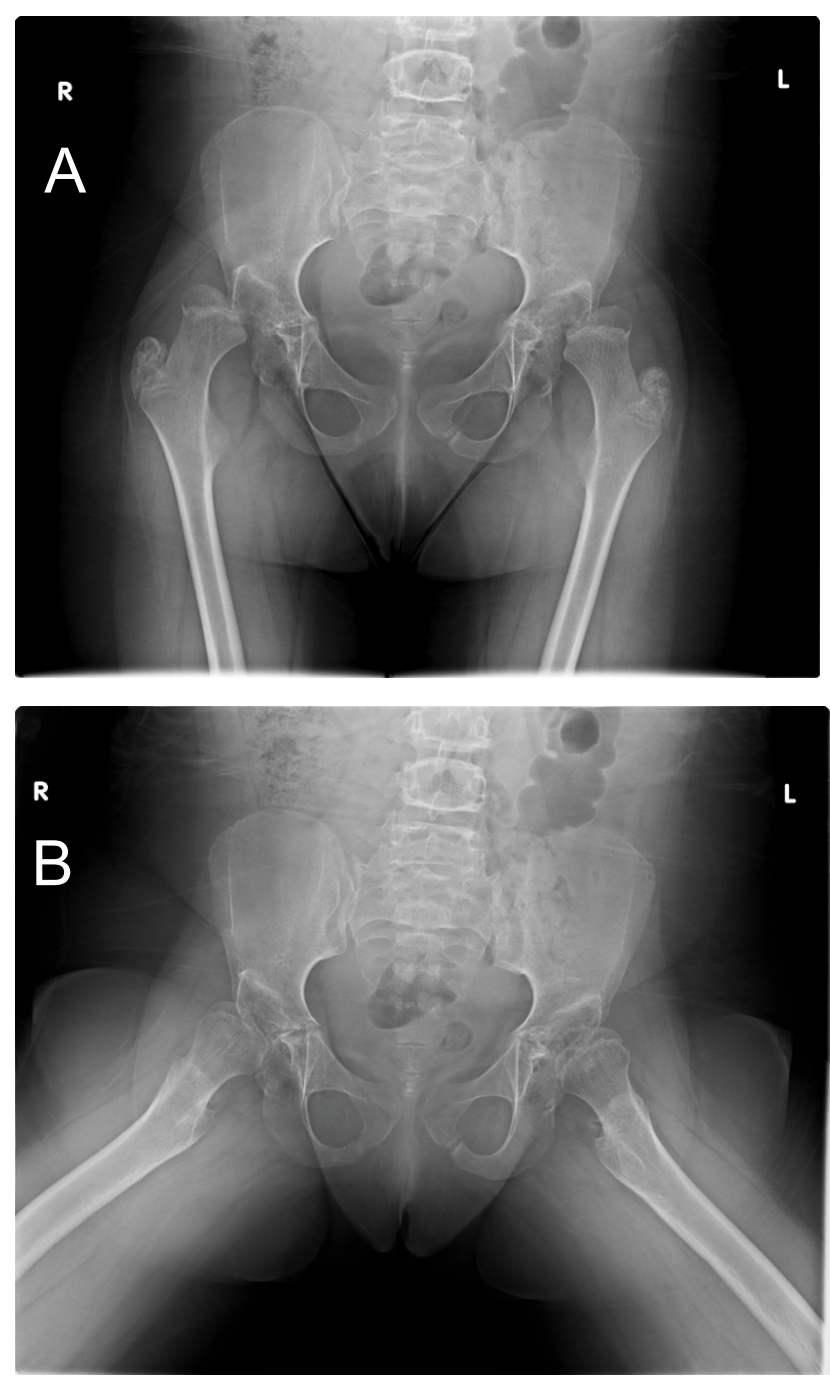

Figure 1A: Stand AP pelvis radiograph demonstrating superolateral escape of the femoral head, coxa valga, femoral head deformity, and severe acetabular dysplasia.

Figure 1B: Frog pelvic radiograph demonstrating an aspherical femoral head.

hygiene was also becoming more challenging as right leg positioning in abduction was becoming more difficult.

Upon physical examination, the patient had increased pain and resistance to abduction of the right hip; she was able to internally rotate 80 degrees. An extensive workup was also performed, which included an MRI scan of the patient's lumbar spine and a CT scan of the patient's pelvis. The MRI scan showed no indications of neurologic causes for her pain. X-rays were obtained (Figure 1) demonstrating bilateral hip dysplasia with Reimer's Migration Percentage (RMP) of 90 right and 80 left, coxa valga and femoral head deformities. There were corresponding superolateral osteoarthritis changes from the abnormal articulation between the head and lip of the acetabulum.

To address our patient's late stage presentation, we decided to perform a Chiari osteotomy plus a shelf procedure. Unlike other operations where the acetabular cartilage and the subchondral bone are redirected, the Chiari medial displacement osteotomy allows for the use of cancellous bone from the ilium $[17,18]$ along with the interposed hip joint capsule, to contain the femoral head for weight bearing. This alleviates the patient's source of pain from neuromuscular hip dysplasia and provides the patient with the ability to ambu- late. While Chiari osteotomies are often performed in older patients with subluxation hips for whom no other reconstructive procedures are possible, they have also been shown to be an effective treatment option for young patients with osteoarthritic changes in dysplastic hips [19].

To substantiate the Chiari osteotomy, we decided to perform an adductor tenotomy, an iliopsoas tenotomy, and a tenotomy of the direct and indirect head of the rectus femoris. These procedures would eliminate the potential restriction the tautness of these muscles might have had on hip range of motion and optimal access to the hip bone for performing the Chiari osteotomy. A Varus de-rotational osteotomy would help situate the good super lateral portion of the femoral head into a better biomechanical position for weight bearing; this would be key to optimizing our patient's likelihood for ambulation. With the Chiari osteotomy likely being unable to give proper coverage to the posterior portion of the femoral head, a shelf operation was performed.

The procedure was performed under general and epidural anesthesia in the supine position. To begin, a right hip arthrogram was performed, revealing a deficient superolateral acetabular coverage and increased medial widening. The femoral head also displayed slight deformity with osteoarthrosis of the medial superior aspect. First, right adductor tenotomy was performed.

A Varus derotational osteotomy was performed in the intertrochanteric region with a proximal femoral locking plate because of the patient's increased femoral anteversions and coxa valga. Guide pins were used in the implant and the distal Diaphysis to estimate the degree of derotation. Our goal was to have her patella in neutral alignment in the supine position on the table. This positioning allowed for a straight vertical tracking of the patella.

Through an ilioinguinal approach, the external oblique musculature was elevated and the iliac Apophysis was split. Subperiosteal exposure of the inner and outer tables was performed. The abductor musculature was cleared from the outer hip capsule posteriorly to the sciatic notch. Through the Smith-Peterson interval, both the direct and indirect heads of the rectus femoris were tenotomized. The interval between the rectus femoris and the iliopsoas was identified and developed to allow for access to the medial aspect of the hip joint.

A liner supraacetabular osteotomy was performed from the superior lateral margin of the cotyloid fossa toward the sciatic notch. The superior aspect of the capsule was sharply removed from its insertion along its entire attachment of the acetabulum. This allowed visualization into the hip joint. A Kerrison rongeur was used to make a bicortical osteotomy low on the wall of the sciatic notch. A Gigli saw was passed subperiosteally through the sciatic notch. To facilitate desired exit of the osteotomy through the medial wall of the acetabulum, osteotomes were used to score the bone from lateral to medial, directed 10 degrees cephalad toward the medial acetabular margin. The capsule was tagged with a non-absorbable suture, and tightly interposed against the roof of the dome of the acetabulum through drill holes.

Acetabular displacement of the distal fragment was performed by abducting the hip and applying a medially directed force. Medialization was visualized on fluoroscopy to gauge how much desired correction was necessary. A $2 \mathrm{~cm} \times 3 \mathrm{~cm}$ strip of unicortical (outer table) ilium was harvested via a straight osteotome for shelf augmentation; this was felt to be necessary because of the lack of anterolateral coverage from the Chiari osteotomy. This was plastically deformed and sutured to the cephalad aspect of the lateral capsule. Bone graft was then placed along the outer aspect of the ilium. The osteotomy was fixed with four $3.5 \mathrm{~mm}$ fully threaded stainless-steel screws. 

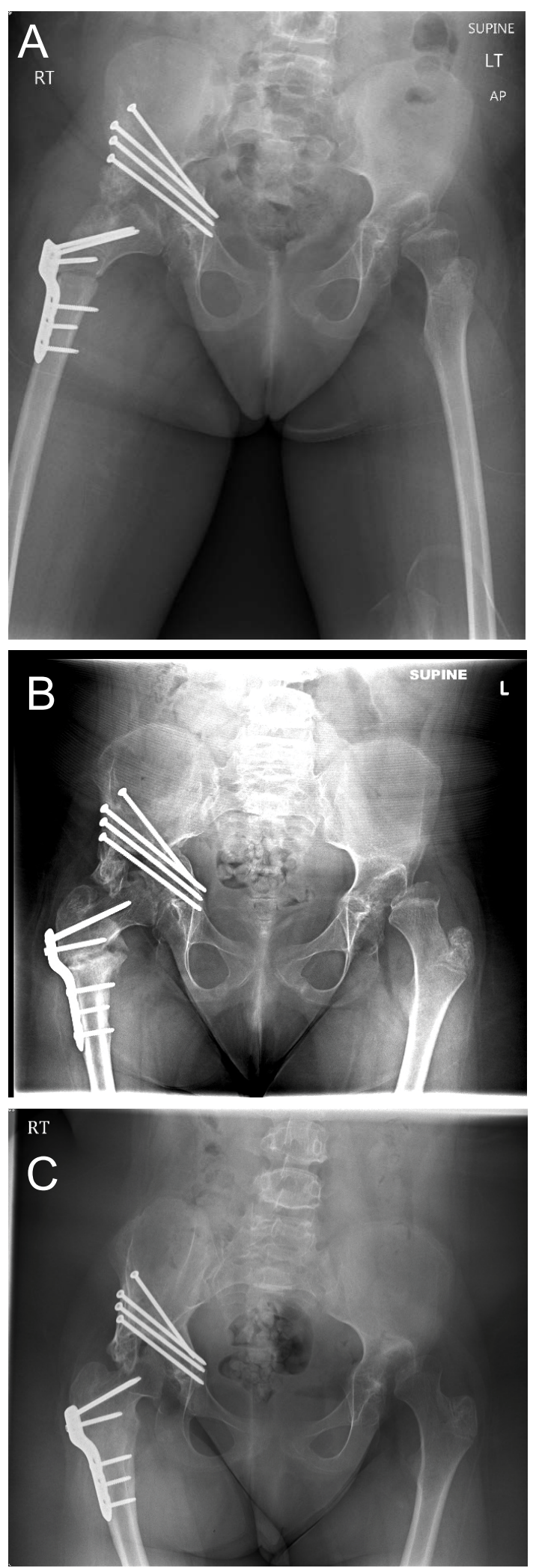

Figure 2A: Stand AP pelvis radiograph demonstrating a healed osteotomy with complete femoral head coverage at two weeks post-op.

Figure 2B: Stand AP pelvis radiograph demonstrating a healed osteotomy with complete femoral head coverage at six months post-op.

Figure 2C: Stand AP pelvis radiograph demonstrating a healed osteotomy with complete femoral head coverage at three years post-op.
A hinged abduction brace was used postoperatively for two weeks, in a position of 30 degrees abduction and 10-80 degrees flexion. Thereafter, weight bearing and physical therapy were initiated.

At six-month follow-up, the patient was using the stander and ambulating unassisted for household distanced around the house. There were no self or proxy reports of pain and (flexed/extended) hip motion was 45 degrees of internal rotation and 50 degrees external rotation. Plain radiographs (Figure 2) at two-weeks, six-months, and three years' post-op follow-up indicate a healed osteotomy with complete femoral head coverage. At the three-year follow-up, the patient continued to be able to stand and transfer, ambulated with assistance, but remained more sedentary.

\section{Discussion}

Before frank dislocation or articular congruity has developed, neuromuscular hip subluxation can be treated with femoral +/- pelvic osteotomy, or soft-tissue procedures [20]. In the setting of incongruity, other salvage options may be considered. Proximal femoral resections have been shown to increase ROM, improve perineal hygiene, increase tolerance of sitting position, and significantly reduce pain levels $[3,21]$. A study on the outcomes of 38 hips treated with Girdlestone resection arthroplasty after having failed total hip arthroplasty, exhibited $66 \%, 34 \%$, and $76 \%$ minor, major, and overall complications, respectively. In addition, an increasing number of comorbilities in patients predicted a statistically significant higher reoperation rate and mortality rates [22].

One such potential complication is an inability to ambulate independently. Based on the patient and family's desire to maintain ambulatory ability if possible, proximal femoral resection was not pursued.

Unlike other pelvic osteotomies wherein the acetabular cartilage and subchondral bone are redirected, the Chiari medial displacement osteotomy utilizes the cancellous ilium and interposed hip joint capsule to contain the femoral head for potential weight bearing (Figure 3). An analysis of mid-to long-term outcomes of 163 patients whom underwent Chiari osteotomy demonstrated a 30year survival rate of $78 \%$ and $91.8 \%$ when performed at pre- or early arthritic stages. For patients with advanced osteoarthritis, results demonstrated a 30 -year survival rate of $49 \%$ and $43.6 \%$, respectively [19].

Our surgical technique involved a medial approach to the Chiari osteotomy, and integrated an adductor tenotomy, an iliopsoas tenotomy, a direct and indirect head of rectus femoris tenotomy, a Varus derotational femoral osteotomy, and a shelf augmentation. This was determined to be the best line of surgical treatment as it would provide complete coverage to the femoral head, would improve hip range of motion, would decrease pain levels caused by the patient's hip dysplasia and osteoarthritis, and would place the femoral head into an optimal position for weight bearing (Figure 4). The Chiari osteotomy with shelf augmentation provides adequate anterolateral acetabular coverage but a potential disadvantage of such a procedure is that it may be unstable to drastically alter posterior femoral head coverage given the angle of the osteotomy (Figure 3).

To address such a possibility, medial displacement of the hemi pelvis may serve to deepen the acetabulum. However, some patients may still necessitate more correction. If necessary, the shelf bone grafting should be placed with this in mind, to help augment any posterior deficiencies and to maintain the femoral head coverage this procedure aims to provide.

At three years of follow-up with minimal complications directly related to our surgical procedure, we believe our surgical technique in performing the Chiari osteotomy was a successful and an effective 


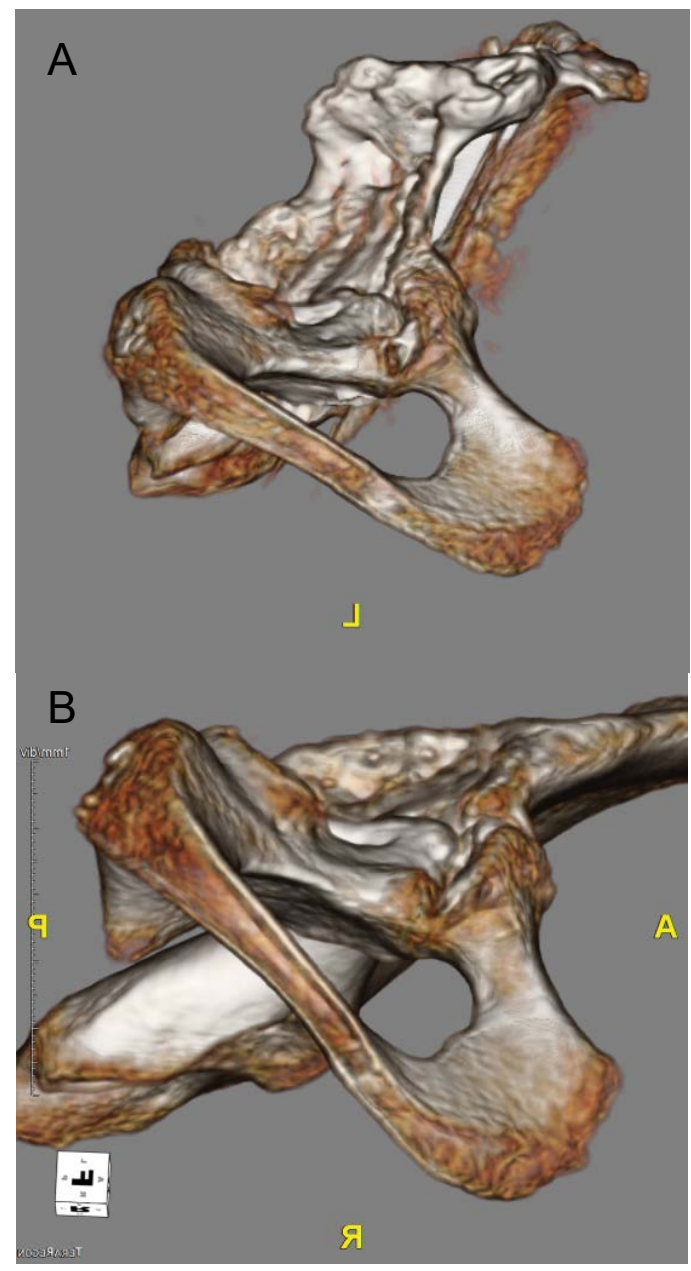

Figure 3A: 3-D CT of a lateral view comparison of superolateral acetabular coverage of the operative right hip.

Figure 3B: 3-D CT of a lateral view comparison of superolateral acetabular coverage of the non-operative left hip. The Chiari osteotomy with shelf augmentation improves the superolateral coverage of the hip but does not fully correct posterior acetabular deficiencies.

means of treating neuromuscular hip dysplasia. We propose that a Chiari plus shelf is an available treatment modality for ambulatory neuromuscular patients with hip subluxation but advanced head deformity precluding reconstruction.

\section{Conflicts of Interest}

There are no conflicts of interest to report.

\section{References}

1. Bascarevic Z, Slavkovic N, Spasovski D, et al. (2011) Chiari pelvic osteotomy in the treatment of adolescent hip disorders: Possibilities, limitations and complications. International Orthopaedics 35: 1203-1208.

2. Kotz R, Chiari C, Hofstaetter J, et al. (2009) Long-term experience with chiari's osteotomy. Clinical Orthopaedics and Related Research 467: 2215-2220.

3. Kovanda M, Rozkydal Z (2003) Chiari pelvic osteotomy in the management of developmental hip dysplasia: A long term follow-up. Bratisl Lek Listy 104: 7-13.

4. Karami M, Fitoussi F, Ilharreborde B, et al. (2008) The results of
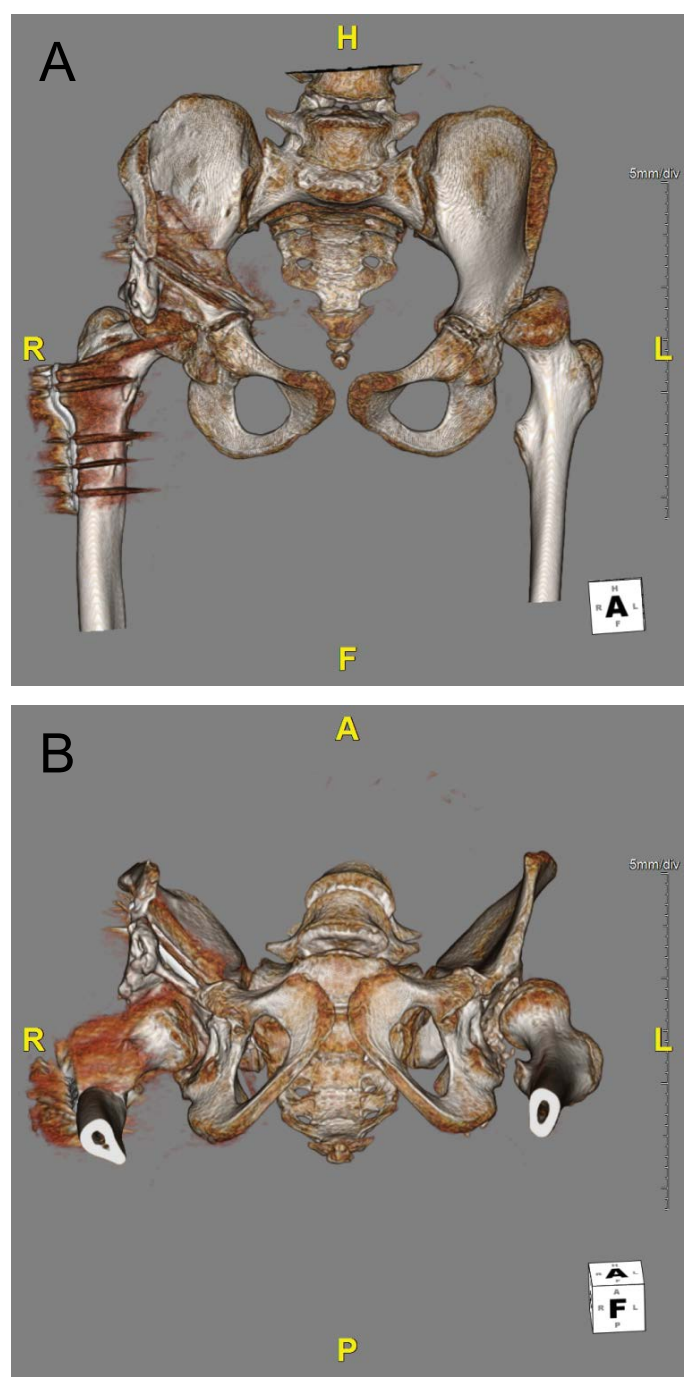

Figure 4A: 3-D CT pelvic of an AP view demonstrating improved femoral head coverage in comparison to the left side.

Figure 4B: 3-D pelvic of an Axial view demonstrating improved femoral head coverage in comparison to the left side.

chiari pelvic osteotomy in adolescents with a brief literature review. Journal of Child Orthopaedics 2: 63-68.

5. Baily Te, Hall Je (1985) Chiari medial displacement osteotomy. Journal of Pediatric Orthopaedics 5: 635-641.

6. Li L, Jia J, ZhaoQ, et al. (2012) Evaluation of femoral head coverage following chiari pelvic osteotomy in adolescents by three-dimensional computed topography and conventional radiography. Archives of Orthopaedic and Trauma Surgery 132: 599-605.

7. Klaue K, Sherman M, Perren SM, et al. (1993) Extra-articular augmentation for residual hip dysplasia. Journal of Bone Joint Surgery-Britain 75: 750-754.

8. Fong HC, Lu W, Li YH, et al. (2000) Chiari osteotomy and shelf augmentation in the treatment of hip dysplasia. Journal of Pediatric Orthopaedics 20: 740-744.

9. Bakarman KA (2013) New technique to overcome surgical pitfalls during chiari osteotomy. Journal of Taibah University Medical Sciences 8: 123-125.

10. Hogh J, MacNicol MF (1987) The chiari pelvic osteotomy: A longterm review of clinical and radiographic results. Journal of Bone Joint Surgery-Britain 69: 365-373. 
11. Colton CL (1972) Osteotomy for acetabular dysplasia in young subjects. Journal of Bone Joint Surgery Britain 54: 578-589.

12. Moll FK Jr (1982) Capsular change following chiari innominate osteotomy. Journal of Pediatric Orthopaedics 2: 573-576.

13. Galuppi B, Palisano R, Rosenbaum P, et al. (1997) Development and reliability of a system to classify gross motor function in children with cerebral palsy. Developmental medicine and child neurology 39: 214-223.

14. Chester Jr, Eulenberg J, Evatt M, et al . (2011) Developing and Validating the Communication Function Classification System for Individuals with Cerebral Palsy. Developmental Medicine and Child Neurology 53: 704-710.

15. Bauer MD. Antithrombin Deficiency. Post TW, ed. UpToDate. Waltham, MA.

16. Flynn J, Spiegel D, (2006) Evaluation and treatment of hip dysplasia in cerebral palsy. Orthopedics Clinics of North America 37: 185-196.

17. Chiari K (1974) Medial displacement osteotomy of the pelvis. Clinical Orthopaedics 98: 55-71.
18. Damla H, Kalchschmidt K, Luering C, et al. (2019) Risk factors associated with non-union after triple pelvic osteotomy (Toennis \& Kalchschmidt Technique): A case -control study and review of the literature. Archives of Orthopaedic and Trauma Surgery 139.

19. Ito H, Matsuno T, Minami A, et al. (2018) The chiari pelvic osteotomy for patients with dysplastic hips and poor joint congruency: Long-term follow-up. The Bone and Joint Journal 93-B 6.

20. Bagg M, Mchale K, Nason S, et al. (1990) Treatment of the chronically dislocated hip in adolescents with cerebral palsy with femoral head resection and subtrochanteric valgus osteotomy. Journal of Pediatric Orthopedics 10: 504-509.

21. Ackerly S, Olney B, Rockley B, et al . (2003) Proximal femoral resection for subluxation or dislocation of the hip in spastic quadriplegia. Developmental Medicine \& Child Neurology 45: 436-440.

22. Elsharkawy KA, Gad BV, Higuera CA, et al. (2015) Complication, survival, and reoperation rates following girdlestone resection arthroplasty. Journal of Arthroplasty 30: 1183-1186.

23. Gibson N, Graham H, Kentish M, et al. (2014) Australian hip surveillance for children with cerebral palsy. Australian academy of cerebral palsy and developmental medicine. 